University of Nebraska - Lincoln

DigitalCommons@University of Nebraska - Lincoln

Faculty Publications, Department of Physics and Astronomy

Research Papers in Physics and Astronomy

1994

\title{
Simplest Atomic System for Sub-Doppler Laser Cooling
}

R. Gupta

SUNY Stony Brook

S. Padua

SUNY Stony Brook

C. Xie

SUNY Stony Brook

Herman Batelaan

University of Nebraska - Lincoln, hbatelaan@unl.edu

Harold Metcalf

SUNY Stony Brook, harold.metcalf@stonybrook.edu

Follow this and additional works at: https://digitalcommons.unl.edu/physicsfacpub

Part of the Physics Commons

Gupta, R.; Padua, S.; Xie, C.; Batelaan, Herman; and Metcalf, Harold, "Simplest Atomic System for SubDoppler Laser Cooling" (1994). Faculty Publications, Department of Physics and Astronomy. 123. https://digitalcommons.unl.edu/physicsfacpub/123

This Article is brought to you for free and open access by the Research Papers in Physics and Astronomy at DigitalCommons@University of Nebraska - Lincoln. It has been accepted for inclusion in Faculty Publications, Department of Physics and Astronomy by an authorized administrator of DigitalCommons@University of Nebraska Lincoln. 


\title{
Simplest atomic system for sub-Doppler laser cooling
}

\author{
R. Gupta, S. Padua, C. Xie, H. Batelaan, and H. Metcalf \\ Department of Physics, State University of New York at Stony Brook, Stony Brook, New York 11790
}

Received August 19, 1993

\begin{abstract}
Sub-Doppler laser cooling requires optical pumping among differently light-shifted ground-state sublevels. We describe a study of the simplest possible angular-momentum configuration that permits all sub-Doppler cooling phenomena. The $J_{g}=1 \Rightarrow J_{e}=0$ angular-momentum configuration shows recoil-limited cooling in the two most well-known types of polarization gradient, magnetically induced laser cooling, velocity-selective resonances, transient cooling, and velocity-selective population trapping.
\end{abstract}

PACS numbers: $32.80 . \mathrm{Pj}, 42.50 . \mathrm{Vk}$

\section{INTRODUCTION}

In all cases of sub-Doppler laser cooling (SDLC) the role of multiple ground-state sublevels, including coherences between them established by either Raman or Zeeman processes, is of paramount importance. The natural question is, What is the simplest possible atomic-level system that would permit SDLC? Since multiple groundstate sublevels are required for SDLC, a four-level $0 \Rightarrow$ $1\left(J_{g} \Rightarrow J_{e}\right)$ transition will not work, but $J=1 \Rightarrow 0$ and $1 / 2 \Rightarrow 1 / 2$ transitions will both work. However, the $1 / 2 \Rightarrow 1 / 2$ scheme will not permit $\sigma^{+}-\sigma^{-}$polarizationgradient cooling, because this requires atomic alignment, ${ }^{1}$ so $1 \Rightarrow 0$ is the simplest possible general scheme for SDLC.

We have studied SDLC in the $F=1 \Rightarrow 0$ transition of the $28 \%$ abundant ${ }^{87} \mathrm{Rb}$ isotope in a one-dimensional (1-D) optical molasses, with and without polarization gradients, and in weak and strong magnetic fields (strong means Zeeman splitting larger than the optical pumping rates). The most important characteristic of this transition in light of either linear or circular polarization is the presence of two unexcitable ground-state sublevels (dark states) as shown in Fig. 1. Effective laser cooling depends on there being many scattering events, because the momentum exchange from light scattering is typically much smaller than the momentum of thermal atoms. In the usual $J \Rightarrow J+1$ transition schemes, atoms are optically pumped into a cyclic transition that permits this required multiple scattering, rather than into a dark state that precludes it. Thus laser cooling on the $F=1 \Rightarrow 0$ transition is quite different from the more common experiments, because transient processes preclude steadystate cooling forces ${ }^{2,3}$ and there is no Doppler cooling. On the other hand, there is both transient laser cooling and velocity-selective population trapping (VSPT).

For the $F=1 \Rightarrow 0$ transition in a 1-D field with no polarization gradients, there are two dark states that are independent of atomic velocity. However, with counterpropagating beams of different polarizations $\epsilon_{1}$ and $\epsilon_{2}$, for example, circular and linear, there is only one velocityindependent dark state. The other two atomic groundstate sublevels combine to form a second state that is dark only at $v=0$ in a semiclassical picture and a third state that is readily excited. This $v=0$ dark state in a 1-D light field with a polarization gradient is a new discovery and is discussed below. VSPT in this dark state of the 1-D optical field can be eliminated only with a strong $\mathbf{B}$ field that is not parallel to either $\boldsymbol{\epsilon}$ for linear polarization or to $\mathbf{k}$ for circular polarization. One can easily understand the necessity of such a $\mathbf{B}$ field by considering that the $\epsilon$ 's have no components parallel to $\mathbf{k}$, and if $\mathbf{k}$ were chosen as the $z$ axis then there would be no optical pumping from the $M_{F}=0$ sublevel in the absence of a $\mathbf{B}$ field to $\mathrm{mix}$ the states.

We note three special features of the $F=1 \Rightarrow 0$ transition. First, steady-state processes are easier to study, since nonsteady-state effects may be present for a much longer time for larger $F_{g}$ values. For example, in circularly polarized light, optical pumping to the steady-state population distribution among the $M_{F}$ sublevels occurs before steady-state cooling on a cyclic transition begins. ${ }^{3}$ Second, calculations of the damping force or the velocity distribution are simplified because of the relatively small Hamiltonian matrix. Third, $F_{e}=0$ requires that the ground state can be optically coupled to only one excited state so that ground-state coherences are not diluted by multiple excited states.

In Section 2 we present a detailed discussion of several special phenomena of laser cooling in the $F=1 \Rightarrow 0$ transition. In Section 3 we describe our apparatus and present the experimental results, including observation of VSPT. In Section 4 we summarize our findings.

\section{SUB-DOPPLER COOLING ON}

AN $F=1 \Rightarrow 0$ TRANSITION

The special characteristics of laser cooling on an $F=$ $1 \Rightarrow 0$ transition can be used to elucidate many features of SDLC. We first consider laser cooling in either the $\sigma^{+}-\sigma^{-}$or the lin $\perp$ lin configurations ${ }^{4}$ with a strong $\mathbf{B}$ field applied perpendicular to the optical $\mathbf{k}$ vectors (for the lin $\perp$ lin case, $\mathbf{B}$ must not be parallel to either of the polarizations $\epsilon$ ). In these cases each traveling-wave laser beam can induce both $\pi$ and $\sigma$ transitions in the frame with the quantization axis chosen along the strong magnetic field. Then both polarization schemes permit an energy-exchange-based description of laser cooling toward $v=0$ (Sisyphus cooling). 

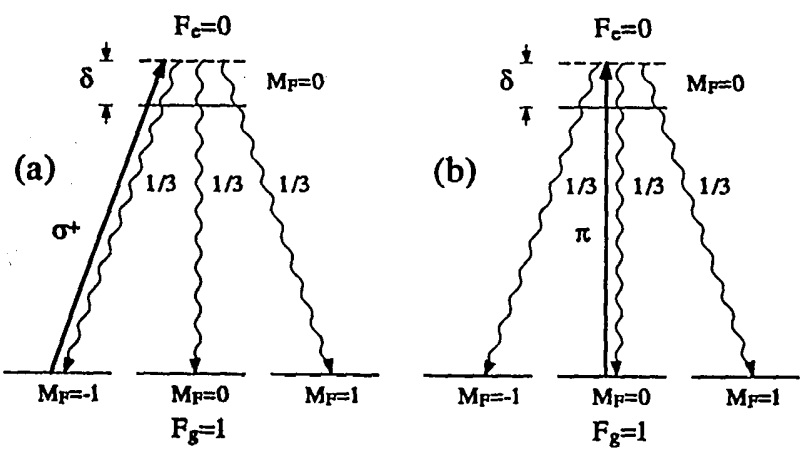

Fig. 1. Energy-level scheme for an $F=1 \Rightarrow 0$ transition, showing allowed excitation for blue-tuned (a) $\sigma^{+}$light and (b) $\pi$ light. In (a) the $M_{F}=0$ and $M_{F}=1$ states are dark, whereas in (b) the $M_{F}= \pm 1$ states are dark. Linearly polarized light perpendicular to $\mathrm{z}$ would excite both the $M_{F}=+1$ and $M_{F}=-1$ sublevels, leaving $M_{F}=0$ as one dark state, while an uncoupled superposition of the $M_{F}= \pm 1$ states would be the other.

To understand this description, we view the optical field in either polarization scheme as two orthogonal, linearly polarized standing waves with nodes spatially displaced from one another as shown in Fig. 2(a). Such a field is symmetric for rotation about $\mathbf{k}$, so we choose one of the $\epsilon$ 's along $\mathbf{B}$. Thus one standing-wave field induces $\pi$ transitions and the other induces $\sigma$ transitions.

We consider light of sufficiently low intensity and large detuning that the excited-state population is small. Atoms are then subject to the conservative force from the light-shift potential (calculated by adiabatic elimination of the excited state). We consider the conservative motion of the atoms in the sinusoidal light-shift potentials, coupled with the optical pumping that provides the irreversibility required for production of damping forces on the atomic motion, as shown schematically in Fig. 2(b). Note that the strengths of the $\sigma$ transitions are smaller than those of $\pi$ transitions for the same amplitude of linear polarization $|\epsilon|$, so the potential hills are correspondingly smaller. Since the potential hills associated with the light shift for the standing wave of one polarization are displaced from those of the other, optical pumping can switch the atomic populations among the ground-state sublevels so that moving atoms always climb the potential hills. ${ }^{5}$

This is indeed what happens when the detuning from atomic resonance $\delta \equiv \omega_{\text {laser }}-\omega_{\text {atom }}>0$. Atoms that enter the light field near an antinode of standing wave $\epsilon_{1}$ are quickly optically pumped out of the sublevel excited by $\epsilon_{1}$. Such atoms cannot be excited by $\epsilon_{2}$ because they are at the node for this polarization, where the excitation rate is zero (as is the light shift). Their transverse velocity carries them toward the antinode of $\epsilon_{2}$, and in the process they increase their internal energy because of positive light shift and correspondingly decrease their kinetic energy and slow down. Near the antinode of $\epsilon_{2}$, where the light intensity and the light shift are largest, the slowed atoms are optically pumped into the state excited by $\epsilon_{1}$, but they are now at the node of this standing wave. The energy-loss process repeats, and the atomic sample is cooled.

In contrast to the energy-based Sisyphus picture described above, laser cooling in these polarization configurations can also be viewed in the momentum-based, velocity-selective resonance (VSR) picture. In this case we necessarily choose a description of the light field as counterpropagating traveling waves instead of standing waves. When $\mathbf{B}$ is strong enough for the Zeeman splitting to be larger than the optical pumping rate $\gamma_{p}$, thereby making $\mathbf{B}$ a suitable choice for the quantization axis, each light beam (not each standing wave) in either the $\sigma^{+}-\sigma^{-}$or the lin $\perp$ lin configuration can induce both $\pi$ and $\sigma$ transitions. Thus there can be VSR, comprising excitation by one beam followed by stimulated emission by the other, back to the original ground-state sublevel as shown by the dashed arrows in Fig. 3. Such a sequence can produce strong velocity damping toward the resonance velocity, which is $v=0$ in this case, because the initial and the final states have the same energy ${ }^{6,7}$ (same state).

This VSR picture provides insight beyond the Sisyphus picture because there are also VSR's between pairs of sublevels of different energies. These are levels that are

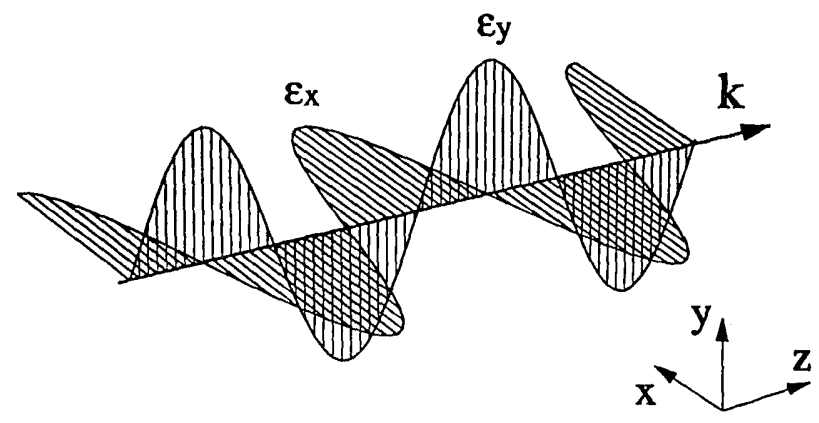

(a)

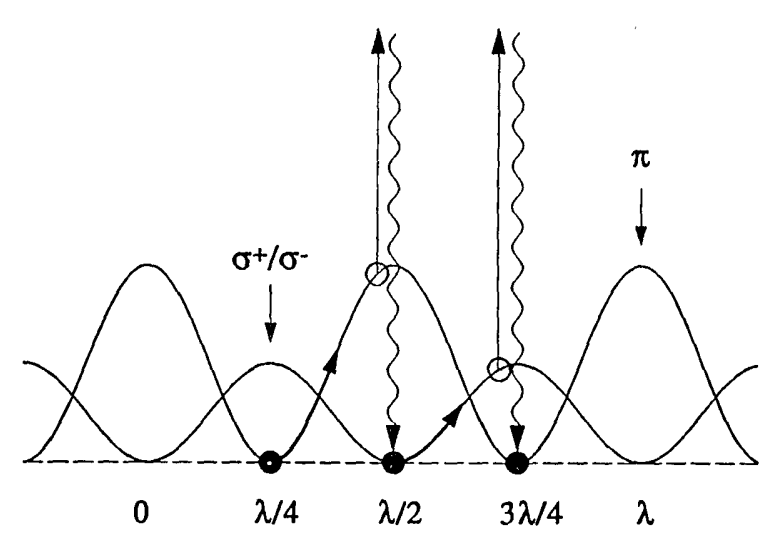

(b)

Fig. 2. (a) Standing-wave fields of either a $\sigma^{+}-\sigma^{-}$or a lin $\perp$ lin polarization configuration. Both optical fields have the same $\lambda / 4$ spatial phase shift. The only difference between the two polarization schemes is the relative temporal phase of the linearly polarized standing waves. This phase difference is zero for $\sigma^{+}-\sigma^{-}$and $\pm \pi / 2$ (i.e., the time lag of $\pm \pi / 2 \omega_{\text {laser }}$ ) for $\operatorname{lin} \perp$ lin. The selection rules are the same. If $\mathbf{B}$ is along one of the $\epsilon$ 's in the lin $\perp$ lin case, one standing wave is parallel to the quantization axis along B, so it induces $\pi$ transitions; the other standing wave is perpendicular to $\mathbf{B}$, so it induces both $\sigma^{+}$and $\sigma^{-}$transitions. In the $\sigma^{+}-\sigma^{-}$case, choosing $\mathbf{z}$ along $\mathbf{B}$ yields the same selection rules as the lin $\perp$ lin case (see Ref. 7). (b) Spatial dependence of the light shift for the field of (a) when the quantization axis is chosen perpendicular to $\mathbf{k}$ (at $\pm 45^{\circ}$ to the $\epsilon$ fields for lin $\perp$ lin). Since the strength of the $\pi$ transition is larger, the light shift is larger for the same $|\epsilon|$ 


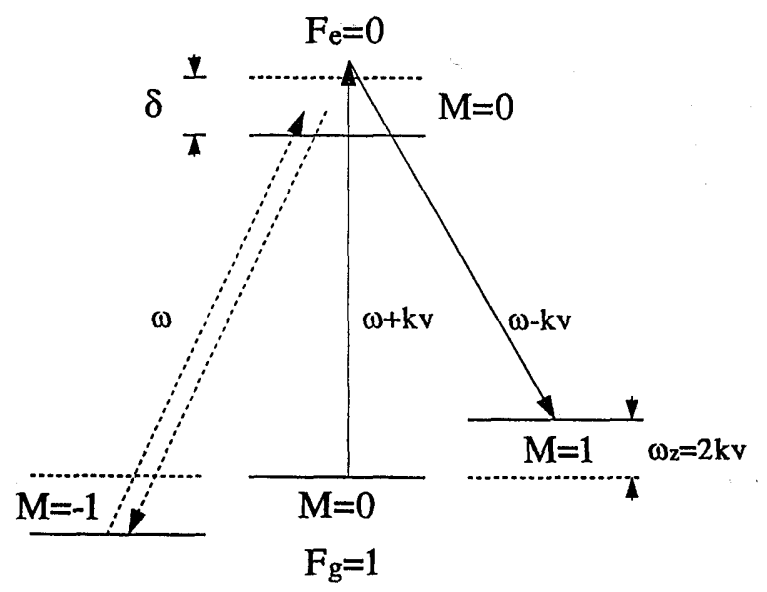

Fig. 3. Raman transitions among the $M_{F}$ sublevels of the $F=1 \Rightarrow 0$ transition in a magnetic field. VSR's at $v=0$ are induced in atoms that return to their original sublevel (dashed arrows) and at finite velocity $v_{r}=\omega_{Z} / 2 k$ for atoms that are transferred between states of different $M_{F}$ (solid arrows).

Zeeman split by $\omega_{Z} \equiv g_{F} \mu_{B} B,{ }^{6,7}$ and Raman transitions between them are shown by the solid arrows in Fig. 3 . Such Raman transitions are resonant when $2 \mathbf{k} \cdot \mathbf{v}= \pm \omega_{Z}$ and are resolvable when $\gamma_{p}<\omega_{Z}$. Our data presented below show strong sub-Doppler cooling to both $v=0$ and $v= \pm \omega_{Z} / 2 k$.

\section{EXPERIMENT}

\section{A. Experimental Apparatus}

Most of our experimental setup has been described in previous work ${ }^{6,8}$ and is only briefly described here. $\mathrm{Rb}$ atoms emerge from a $150^{\circ} \mathrm{C}$ oven with a horizontal slit aperture $0.06 \mathrm{~mm}$ high by $2 \mathrm{~mm}$ wide (see Fig. 4). A horizontal atomic beam is formed by a vertical slit $2 \mathrm{~mm}$ high by $0.06 \mathrm{~mm}$ wide, $35 \mathrm{~cm}$ away from the oven. The atomic beam is crossed at $90^{\circ}$ by a pair of counterpropagating, horizontal laser beams just beyond the vertical slit, and the polarization of the retroreflected beam can be modified by a quarter-wave plate in front of the mirror.
Three pairs of square Helmholtz coils are used for controlling the $\mathbf{B}$ field in the interaction region. The atomicbeam profile parallel to the direction of the optical $\mathbf{k}$ vectors is measured with a scanning hot platinum-tungsten wire, $25 \mu \mathrm{m}$ in diameter, $1.3 \mathrm{~m}$ away from the interaction region.

A 20-mW Sharp Model LTO24 diode laser is sidelocked to a Doppler-broadened signal from a $\mathrm{Rb}$ cell at room temperature. Light from this laser is used to optically pump the ${ }^{87} \mathrm{Rb}$ atoms from the $5 S_{1 / 2}(F=2)$ to the $5 S_{1 / 2}(F=1)$ ground hyperfine structure state before they reach the interaction region.

Light from a second diode laser, a 35-mW Sharp Model LTO25, is split into two beams, and the weaker one passes through an $80-\mathrm{MHz}$ acousto-optic modulator (AOM) and then to another $\mathrm{Rb}$ vapor cell. A crossover resonance in its saturated absorption signal is used to lock the laser near the $F=1 \Rightarrow 0$ transition in ${ }^{87} \mathrm{Rb}$ near $\lambda=780 \mathrm{~nm}$, and the AOM facilitates tuning on either side of the atomic resonance. Approximately $10^{-5}$ of the laser light is fed back to the laser by a blazed grating (see Fig. 4) to reduce the spectral width to $\leq 1 \mathrm{MHz} .{ }^{9}$ The laser beam has its spatial intensity profile flattened to a few percent by a tilted étalon ${ }^{10}$ and is then expanded to $23 \mathrm{~mm}$ wide by $5 \mathrm{~mm}$ high. A mechanical shutter interrupts this beam, and many systematic effects have been eliminated by subtracting data from measurements with the laser beam off from those with the beam on.

\section{B. Experimental Results}

Figure 5 shows the measured atomic-beam profiles with $\mathbf{B}$ perpendicular to the $\mathbf{k}$ vectors for the case of $\sigma^{+}-\sigma^{-}$ [Fig. 5(a)] and lin $\perp$ lin with $\mathbf{B}$ at $45^{\circ}$ to the polarization vectors [Fig. 5(b)]. In each case $\mathbf{B}$ is perpendicular to the horizontal atomic and laser beams. The top trace in each figure shows strong sub-Doppler cooling to $v=0$ and a velocity width of only $\sim 5.5 \mathrm{~cm} / \mathrm{s}$ (Doppler limit approximately $13 \mathrm{~cm} / \mathrm{s}$ in 1-D optical molasses). Both the top traces were taken with large $B(B>1 \mathrm{G})$, and both also show the VSR peaks at $v \neq 0$. As $\mathbf{B}$ is reduced (lower traces), these VSR peaks move to lower velocities,

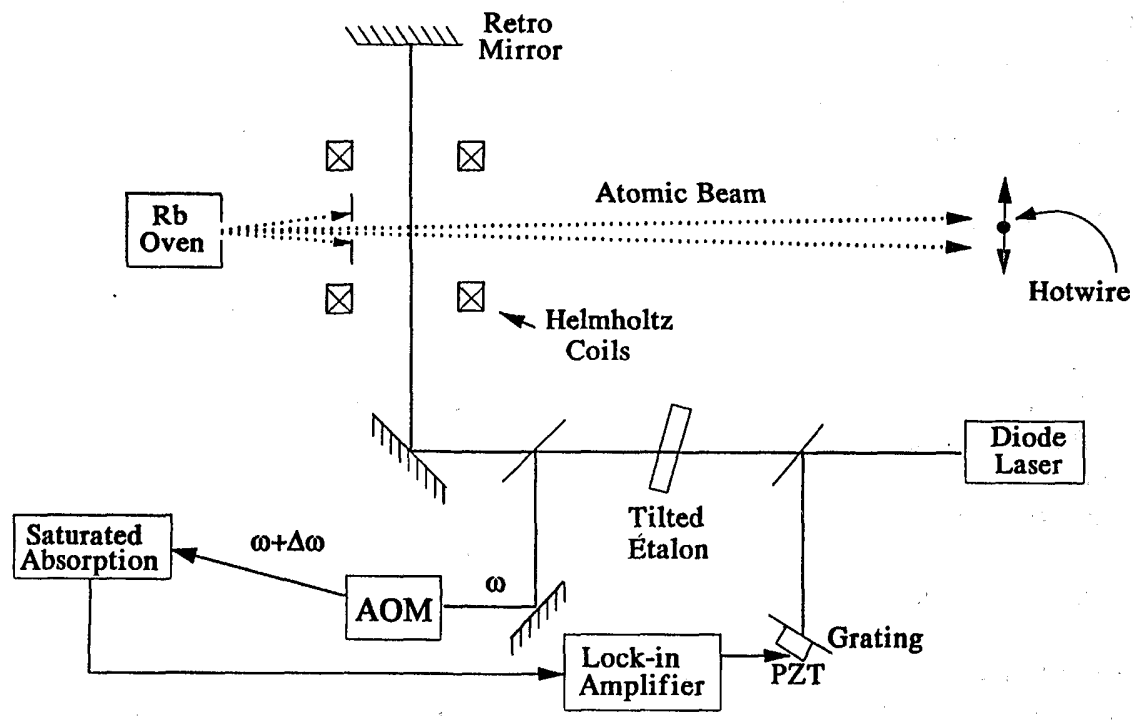

Fig. 4. Schematic diagram of the apparatus; PZT, piezoelectric translator. 


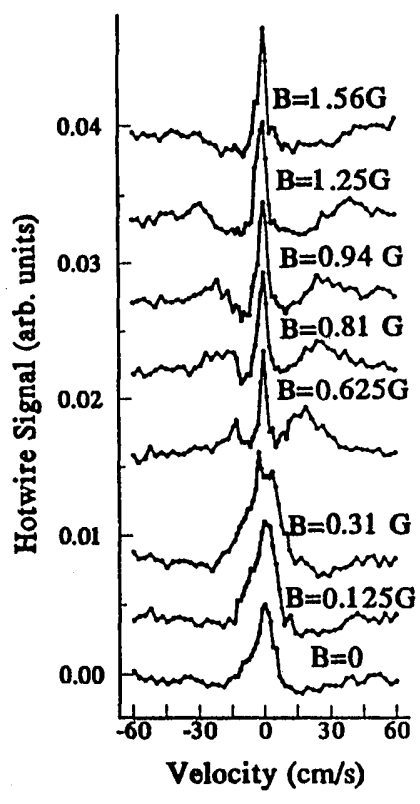

(a)

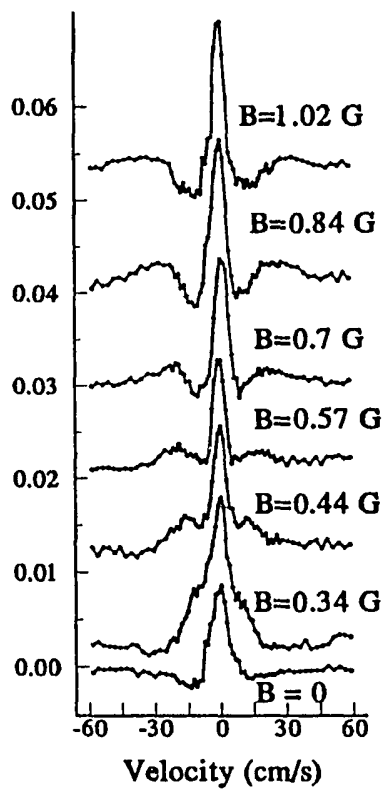

(b)
Fig. 5. Measured atomic-beam profiles for $\delta / 2 \pi=+12 \mathrm{MHz}$ $(\cong 2 \gamma)$ for (a) $\sigma^{+}-\sigma^{-}$with $s=1.33$ and (b) lin $\perp$ lin with $s=0.66$. The highest $\mathbf{B}$ values are at the top, decreasing to $\mathbf{B}=0$ at the bottom. Note that the VSR signals on each side of the central peak are broadened by the longitudinal velocity distribution.

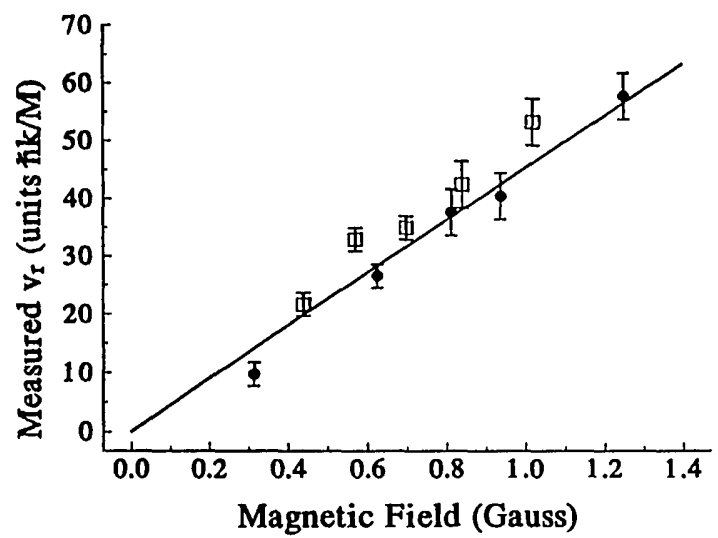

Fig. 6. Velocity of the VSR peaks in Fig. 5 versus B. The filled circles are for $\sigma^{+}$[Fig. 5(a)], and the open squares are for lin $\perp$ lin [Fig. 5(b)]. The straight line represents the resonance case, $v=\omega_{Z} / 2 k$, appropriate in each case because the light induces both $\pi$ and $\sigma$ transitions in the strong transverse $\mathbf{B}$ field.

and Fig. 6 shows the separation of these peaks versus $|\mathbf{B}|$ for each case.

In the semiclassical approximation the order of the velocity capture range of this cooling process is determined by the lifetime of the ground-state populations and/or coherences produced by the VSR's. Atoms are optically excited out of these coherences at a rate $\gamma_{p}=\tilde{\gamma}_{p} \cos ^{2}(k x)$, where $\tilde{\gamma}_{p} \equiv 2 s(\gamma / 2) /(3 L+s), \tau \equiv 1 / \gamma$ is the excited-state lifetime (27 ns for this $\mathrm{Rb}$ transition), $L \equiv 1+(2 \delta / \gamma)^{2}$, and the saturation parameter for a single light beam is $s \equiv I / I_{\text {sat }}$, where $I_{\text {sat }} \equiv \pi h c / 3 \lambda^{3} \tau\left(\equiv 1.6 \mathrm{~mW} / \mathrm{cm}^{2}\right.$ for the $\lambda=780 \mathrm{~nm}$ transition in $\mathrm{Rb})$. The factor of 3 in the denominator of $\tilde{\gamma}_{p}$ comes from the Clebsch-Gordan coefficient for the $1 \Rightarrow 0$ transition for each value of $M_{F}$, and the factor of 2 in the numerator comes from the superposi- tion of two orthogonal light beams. For Fig. 5(a) $s \cong 1.33$ and $\delta \cong 2 \pi \times 12 \mathrm{MHz}$, so that $\tilde{\gamma}_{p} / 2 \pi \cong 150 \mathrm{kHz}$, and the average value over a wavelength $\tilde{\gamma}_{p}$ is half of that.

Under optimum cooling conditions the expected width of the velocity distribution is $\sim \tilde{\gamma}_{p} / k \cong 6 \mathrm{~cm} / \mathrm{s}$, about equal to the value observed for the $v=0$ peak. The widths of the $v \neq 0$ peaks, $\Delta v$, are limited by the width of the longitudinal velocity distribution $\Delta v_{l}$ through $\Delta v / v \sim$ $\left(\Delta v_{l} / v_{l}\right) \sim 1 / 2$ for a thermal beam, and the peaks' widths are indeed approximately half their distances from the $v=0$ peak. The $v \neq 0$ peaks begin to merge into the $v=0$ peak when their velocity is $\sim 10 \mathrm{~cm} / \mathrm{s}$, corresponding to $|\mathbf{B}| \cong 0.3 \mathrm{G}$ for $g_{F}=1 / 2$. Similar conclusions can be drawn from the data of Fig. 5(b). Furthermore, at low B fields some states are only weakly excited, and transient cooling effects begin to appear. ${ }^{2}$

The two bottom traces in Fig. 5, where $|\mathbf{B}|=0$, exhibit the VSPT discussed above. Atoms traveling in either light field of Fig. 5 with nearly zero transverse velocities spend all their time in light of one particular polarization as they ride along a particular phase of the standing wave. For example, in lin $\perp$ lin this polarization may be circular along one path across the standing wave or linear along another path that is $\lambda / 8$ away. In the absence of a $\mathbf{B}$ field the quantization axis is chosen along the local polarization. Atoms are then optically pumped to a dark state of this basis (Fig. 1) and remain uncoupled to the excited state by the light. These slow atoms are subject to VSPT and thus do not experience momentum diffusion. They could therefore display an arbitrarily narrow velocity distribution with a long enough interaction time consistent with the uncertainty principle. (Note that this is not the same as the coherent population trapping of Ref. 11, which depends on the quantized center-of-mass motion of the atoms.) Atoms are cooled to the low velocities appropriate for VSPT by transient polarization gradient cooling, and these atoms form the sub-Doppler peaks of the bottom traces of Fig. 5 with $\mathbf{B}=0$. When atoms traverse either light field of Fig. 5 with a higher velocity, they experience a constantly changing polarization and could thus be pumped out of the velocity-selective dark states of Fig. 1.

We have tested this hypothesis of VSPT at $|\mathbf{B}|=0$ several ways. First, we found that the cold-atom peaks occur over a wide range of detuning, both blue and red, although the peaks are stronger in the blue case because there is residual polarization-gradient cooling to feed the dark state. Second, we used an aperture on the laser beam to shorten the interaction time and found that the cold-atom peaks require a few optical pumping times $1 / \tilde{\gamma}_{p}$ to appear. Third, we note that $\tilde{\gamma}_{p} \cong \gamma / 80$ for the conditions of Fig. 5, so that optical pumping occurs in $\sim 2.5 \mu \mathrm{s}$. VSPT atoms that move less than $\lambda / 4$ in this time will have to wait longer than this time to be pumped out of the dark state because they will not be subject to a different polarization. Thus there is also a cold peak even in the absence of a $\mathbf{B}$ field with a velocity distribution of width $\sim 9 \mathrm{~cm} / \mathrm{s}, \sim 50 \%$ larger than the large $\mathbf{B}$ field peaks, just as is shown in the bottom traces of Fig. 5 .

In addition to all the experiments described above with the two types of polarization gradient, we have also studied SDLC in the $F=1 \Rightarrow 0$ transition in a standing wave of purely circularly polarized light. The presence of a 
weak transverse $\mathbf{B}$ field then produces magnetically induced laser cooling. ${ }^{8}$ At low $\mathbf{B}$ field we observed a very narrow peak near $v=0$, and at higher fields we saw this peak split into two peaks with sub-Doppler widths centered at $v_{r}= \pm \omega_{Z} / 2 k$ just as in previous experiments using more complicated transitions. ${ }^{6}$ We have also observed higher-order VSR's for which the resonance velocity satisfies $v_{r}{ }^{\prime}= \pm \omega_{Z} / 4 k$, corresponding to redistribution of two photons between the two laser beams that form the optical molasses.

\section{CONCLUSIONS}

We have performed several other experiments on this simplest possible SDLC scheme to ensure that it displays all the features that we expect. For example, in the lin $\perp$ lin case with $\mathbf{B}$ along one of the polarization vectors, we find that both cold peaks of atoms indeed move at the resonance velocity $v_{r}= \pm \omega_{Z} / 2 k .{ }^{12}$

Thus we conclude that the $F=1 \Rightarrow 0$ transition is the paradigm of SDLC, displaying many of its special features in addition to microkelvin temperatures and holding claim to being the simplest possible angular-momentum configuration with this capability. In the future we plan more extensive studies of VSPT, the higher-order resonances, and other aspects of this extraordinary laser cooling system.

\section{ACKNOWLEDGMENTS}

This research was supported by the National Science Foundation, the U.S. Office of Naval Research, the U.S.
Air Force Office of Scientific Research, and Coordenadoria de Aperfeicoamento de Estudos e Projetos (Brazil).

\section{REFERENCES}

1. G. Nienhuis, P. van der Straten, and S.-Q. Shang, Phys. Rev. A 44, 462 (1991).

2. S. Padua, C. Xie, R. Gupta, H. Batelaan, T. Bergeman, and H. Metcalf, Phys. Rev. Lett. 70, 3217 (1993).

3. C. Valentin, M.-C. Gagné, J. Yu, and P. Pillet, Europhys. Lett. 17, 133 (1992).

4. J. Dalibard and C. Cohen-Tannoudji, J. Opt. Soc. Am. B 6, 2023 (1989).

5. This presents no conflict with the usual view of $\sigma^{+}-\dot{\sigma}^{-}$ laser cooling ${ }^{1,4}$ because of the strong $B$ field (see Ref. 7). In the usual views where $\mathbf{B}=0$, the damping force cannot be viewed as derived from a Sisyphus mechanism but rather as a scattering force or radiation pressure derived from a population imbalance. Here, however, the strong $\mathbf{B}$ field perpendicular to $\mathbf{k}$ precludes the Larmor transformation responsible for the usual viewpoint. ${ }^{4}$

6. S.-Q. Shang, B. Sheehy, P. van der Straten, and H. Metcalf, Phys. Rev. Lett. 65, 317 (1990).

7. P. van der Straten, S.-Q. Shang, B. Sheehy, H. Metcalf, and G. Nienhuis, Phys. Rev. A 47, 4160 (1993).

8. B. Sheehy, S.-Q. Shang, P. van der Straten, S. Hatamian, and H. Metcalf, Phys. Rev. Lett. 64, 858 (1990).

9. R. E. Ryan, L. A. Westling, and H. J. Metcalf, J. Opt. Soc. Am. B 10, 1643 (1993).

10. C. Xie, R. Gupta, and H. Metcalf, Opt. Lett. 18, 173 (1993).

11. A. Aspect, E. Arimondo, R. Kaiser, N. Vansteenkiste, and C. Cohen-Tannoudji, Phys. Rev. Lett. 61, 826 (1988).

12. S.-Q. Shang, B. Sheehy, H. Metcalf, P. van der Straten, and G. Nienhuis, Phys. Rev. Lett. 67, 1094 (1991). 